\title{
The rights of patients versus the obligations to sponsors in clinical trials
}

\author{
Karl H. Kimbel \\ Ethics Committee for Studies in Human Subjects: North-Rhine Chamber of Physicians, \\ Düsseldorf, Germany \\ (Accepted 1 March 1992)
}

A recent exchange of Letters to the Editor in The Lancet [1,2], has made it evident that the Note for Guidance on "Good Clinical Practice for Trials on Medicinal Products in the European Community" [3], does not provide sufficient advice on the relation between the patient or volunteer on the one hand and the sponsor and his non-physician executive (e.g. monitors) on the other. Although there is no doubt that the patient's interests as represented by the physician have absolute precedence, the latter's responsibilities are listed after those of the sponsor and monitor. Accordingly, two important rights of the patient, i.e. that of the absolute confidentiality of his history and his clinical data as well as of the right to determine with his physician when to terminate his treatment, are insufficiently dealt with.

As has been emphasized by Deutsch et al. [1], the non-physician monitor should, according to the guidelines, "assure...that informed consent is being obtained and recorded from all patients". Since the signature of the patient is to be proof of his consent, the confidentiality is already broken. He should further "check the CRF entries with the source documents", which invariably contain the names of patients at many points, and it is impossible to ensure that all these are blacked out.

The Guidelines put the responsibility for discontinuing the trial squarely with the sponsor $(2.3 \mathrm{c})$. The reasons for discontinuance may relate to the subject's safety but they may equally well be organisational or even commercial. There may be occasions where the abrupt discontinuation of drug treatment may be detrimental to the patient or where the change to a replacement medicine may be not without risk to the patient. In diseases in which the drug on trial may be the first efficacious agent to become available (e.g. erythropoietin) a premature termination

Correspondence to: K.H. Kimbel, M.D., Ethics Committee for Studies in Human Subjects: North Rhine Chamber of Physicians, Tersteegenstrasse 31, D 4000 Düsseldorf 30, Germany. 
of the trial, other than for safety reasons, may also have grave consequences for the patient.

If the contract between the sponsor and the investigator (and subsequently between investigator and patient/volunteer) were at the free discretion of the investigator (or patient) the latter would be free to reject the contractual arrangement in the event of the slightest doubt. The patient and the physician, however, could have an interest in participation in the study. For the patient it may be the hope for more tolerable, more efficacious medicine or simply the wish not to disappoint his physician who has done so much for him. For the physician the interest may range from scientific endeavour or a desire to give what may be a better medication to the attraction of a generous remuneration which may compensate for poor government support of his research work.

Since, as pointed out above, the investigator and volunteer/patient are not completely free to turn down the contractual arrangement nor to protest against any changes introduced during the study, national and supranational guidelines must provide clear-cut guidance on how to avoid commercial interests taking precedence over the acknowledged right of the patient/volunteer to the assurance that his medical and other confidential data will be kept safe by his physician and no-one else, and that he and his physician alone will determine when his treatment be changed or terminated.

There are plans, encouraged too by industry, to elevate the status of the Guidelines on "Good Clinical Practice for Trials on Medicinal Products in the European Community" to a binding Directive. This process may take years, not merely because of the need for consultation but also in order to adopt the scope and wording to the latest state of the art; it may nevertheless not be too early to ask the responsible authorities now to consider seriously the problems discussed above. In the meantime, Ethics Committees should see to it that the protocol of any trial provides for medical personnel bound by strict rules of confidentiality and working as members of the medical team of the trial to read to the monitor such data as he requests in order to avoid divulging to him the full contents of the original documents. The protocol must also specify that the decision as to when the patient's medication (not the trial itself) should be discontinued rests solely with the investigator.

\section{References}

1 Deutsch E, von Eickstedt, KW, Frölich JC, Schneider B. "Monitors" and European Community guideline on clinical trials. Lancet 1991;338:1151.

2 Benbow AG. "Monitors" and EC guidelines. Lancet 1991;338:1598.

3 Commission of the European Communities, Directorate-General for Internal Market and Industrial Affairs: III/3976/88 Final. 